

\title{
PP-waves from rotating and continuously distributed D3-branes
}

\author{
Andreas Brandhuber \\ Department of Theoretical Physics \\ California Institute of Technology \\ Pasadena, CA 91125, USA \\ E-mail: andreas@theory.caltech.edu

\section{Konstadinos Sfetsos} \\ Department of Engineering Sciences, University of Patras \\ 26110 Patras, Greece \\ E-mail: sfetsos@des.upatras.gr
}

ABSTRACT: We study families of PP-wave solutions of type-IIB supergravity that have (light-cone) time dependent metrics and RR five-form fluxes. They arise as Penrose limits of supergravity solutions that correspond to rotating or continuous distributions of D3branes. In general, the solutions preserve sixteen supersymmetries. On the dual field theory side these backgrounds describe the BMN limit of $\mathcal{N}=4$ SYM when some scalars in the field theory have non-vanishing expectation values. We study the perturbative string spectrum and in several cases we are able to determine it exactly for the bosons as well as for the fermions. We find that there are special states for particular values of the light-cone constant $P_{+}$.

Keywords: Penrose limit and pp-wave background, AdS-CFT and dS-CFT Correspondence. 


\section{Contents}

1. Introduction if

2. A class of PP-waves and supersymmetry 2

3. Rotating D3-branes and Penrose limits 5

3.1 Null geodesics 6

3.2 Geodesics at $\theta=\pi / 2$

3.3 Geodesics at $\theta=\pi / 2$ and zero temperature

3.4 Geodesics at $\theta=0$

3.5 Geodesics at $\theta=0$ and zero temperature 10

4. The perturbative string spectrum 11

4.1 Spectra en détail 13

5. Disk distribution in the limit of large radius 14

5.1 PP-wave limit

6. Concluding remarks 16

\section{Introduction}

Recently, an interesting testing ground for the AdS/CFT correspondence was unraveled [1] which allows to make precise predictions and comparisons on both sides of the duality. This is based on the observation that string theory is exactly solvable in particular PPwave backgrounds [2, 3]. Furthermore, these backgrounds are Penrose limits [ [] of the $A d S_{d} \times S^{k}$ space-times, that where intensively studied in the context of the AdS/CFT correspondence. This geometric limit translates to a truncation of the gauge theory to operators of large $\mathcal{R}$ charge. The aim of this paper, with these new insights in mind, is to investigate supergravity backgrounds that have already been used to test the AdS/CFT correspondence successfully. These backgrounds describe deformations of the $\mathcal{N}=4$ supersymmetric Yang-Mills theory by turning on expectation values of scalar fields. The supergravity solutions involve non-trivial RR five-form fluxes and the metric, and they preserve 16 supersymmetries. In the Penrose limit the metric and the five-form depend in general on the light-cone time, unlike the PP-waves with higher supersymmetry, and no supersymmetry enhancement is observed. In addition, we will study backgrounds that correspond to the same Yang-Mills theory at finite temperature and strong 't Hooft coupling which break supersymmetry. In these cases the Penrose limit restores 16 of the broken supersymmetries. 
Besides flat space-time, type-IIB supergravity [6] admits two extra maximally supersymmetric solutions, namely $A d S_{5} \times S^{5}$ [7] and the PP-wave [8], both with the RR five-form flux turned on. The latter solution is the Penrose limit of the former one $\left[0^{1}\right.$ and as shown in [1] string theory in this background is dual to a corner of the $\mathcal{N}=4$ supersymmetric Yang-Mills theory just as the Penrose limit blows up an infinitesimal region around a nullgeodesic in the full $A d S_{5} \times S^{5}$ geometry. Naturally, one is led to the question if this can be generalized to theories with less supersymmetry and/or broken conformal symmetry. Penrose limits of geometries describing RG fixed points have been studied recently which led to $\mathrm{PP}$-waves with various amounts of supersymmetry ranging from 16 to 32 , where in many cases the supersymmetry compared to the original background is enhanced. In all these cases the metric and the fluxes are static (at least after a simple coordinate transformation) and string theory can be solved exactly [12]-[17]. Harder and more interesting is the case of Penrose limits of RG flows which generically lead to PP-waves and fluxes that depend on the light-cone time. These waves always preserve 16 supercharges and generically string theory cannot be solved because the world-sheet theory is interacting. The aim of this paper is to study the Penrose limits of one of the simplest RG flows that can be studied using the AdS/CFT correspondence and investigate aspects of string propagation in the corresponding PP-waves. It turns out that even for these simple models the interactions are quite complicated but in several cases at least the spectrum of string excitations can be worked out explicitly.

This paper is organized as follows: In section 2 we will present the general features of the PP-waves of the form we will discuss later and count the number of supersymmetries they preserve. In section 3 we will take two different Penrose limits on type-IIB backgrounds representing near-extremal rotating D3-branes, or continous distributions of D3-branes and we will describe the resulting PP-waves in detail. Section 4 is devoted to an analysis of the bosonic and fermionic modes of the Green-Schwarz string in these PP-waves. We exhibit cases for which the perturbative string spectra can be found exactly, despite the non-trivial dependence of our backgrounds on the light-cone time. Finally, in section 5 we study D3brane distributions in a limit where the size of the distribution goes to infinity, as well as their Penrose limit. We end the paper with concluding remarks in section 6.

\section{A class of PP-waves and supersymmetry}

Before going into explicit examples let us summarize some basic features of the class of $\mathrm{PP}$-wave solutions that we are going to construct. First of all the dilaton and axion are constant and the Ramond and Neveu-Schwarz two-form gauge fields vanish. The general form of the remaining, non-trivial fields is

$$
d s^{2}=2 d u d v+\sum_{i, j=1}^{8} F_{i j}(u) x^{i} x^{j} d u^{2}+\sum_{i=1}^{8} d x^{i} d x^{i},
$$

\footnotetext{
${ }^{1}$ Penrose limits in order to obtain PP-wave solutions in string theory were first performed in [9], in relation to WZW and gauged WZW models based on non-semisimple groups (initiated in 10 ), and also in [1] for the field equations of the low energy perturbative string theories.
} 


$$
\mathcal{F}_{5}=f(u) d u \wedge\left(1+*_{8}\right)\left(d x_{1} \wedge d x_{2} \wedge d x_{3} \wedge d x_{4}\right),
$$

where $*_{8}$ denotes the Hodge star operator acting on the eight directions transverse to $u, v$. One can easily read off the isometries from (2.1). First there is always a shift symmetry in $v$, whereas a shift of $u$ is a symmetry only if $F_{i j}$ and $f$ are constant. In addition, depending on the $F_{i j}$, there can be symmetries rotating the $x_{i}$. However, the form of the Ramond five-form $\mathcal{F}_{5}$ restricts the maximal symmetry to $\mathrm{SO}(4) \times \mathrm{SO}(4)$ if $F_{11}=F_{22}=F_{33}=F_{44}$, $F_{55}=F_{66}=F_{77}=F_{88}$ and all other $F_{i j}=0$. For less symmetric configurations the symmetry is reduced accordingly. We will not discuss any further, but simply note here, that backgrounds where the space transverse to the light-cone directions is replaced by a curved manifold and the $F_{i j}$ depend only on these transverse coordinates have been studied in 18. 21].

The metric in (2.1) can be written as

$$
d s^{2}=2 e^{+} e^{-}+\sum_{i=1}^{8} e^{i} e^{i}
$$

by introducing the Zehnbeine $e^{a}=e_{M}^{a} d x^{M}$ :

$$
e^{+}=d u, \quad e^{-}=d v+\frac{1}{2} F_{i j} x^{i} x^{j} d u, \quad e^{i}=d x^{i} .
$$

The non-vanishing components of the spin connection $\omega_{a b}$ are

$$
\omega_{+i}=\omega^{-i}=F_{i j} x^{j} d u .
$$

The only non-vanishing components of the Riemann and Ricci tensors are

$$
R_{+i+j}=-F_{i j}, \quad R_{++}=-F_{i}^{i},
$$

simplifying tremendously the classical type-IIB field equations to

$$
-F_{i}^{i}=8 f(u)^{2}
$$

We can determine the number of supersymmetries preserved by the PP-wave solution (2.1) (for $F_{i j}$ and $f$ constants, this was done in [8]). For this purpose we have to set to zero the type-IIB supergravitity variations [6] of the dilatino, $\lambda$, and the gravitino, $\psi_{M}$. For our particular backgrounds the complex three-form vanishes identically, and, therefore, the dilatino equation is trivially solved whereas the gravitino equation reduces to

$$
\delta \psi_{M}=D_{M} \epsilon+\frac{i}{480} \mathcal{F}_{P Q R S T} \Gamma^{P Q R S T} \Gamma_{M} \epsilon,
$$

where $D_{M} \epsilon=\partial_{M} \epsilon+\frac{1}{4} \omega_{M}^{P Q} \Gamma^{P Q} \epsilon$ and we set $\gamma^{11} \psi_{M}=\psi_{M}$, so that $\gamma^{11} \epsilon=\epsilon$.

The covariant derivatives appearing in (2.7) become

$$
D_{-}=\partial_{-}, \quad D_{i}=\partial_{i}, \quad D_{+}=\partial_{+}+\frac{1}{2} F_{i j} x^{j} \gamma_{-} \gamma_{i}
$$


where the $\gamma^{a}$ are ten-dimensional flat space Gamma matrices (for a convenient basis, see, for instance, [3]) and where we have defined

$$
\gamma^{ \pm}=\left( \pm \gamma^{0}+\gamma^{9}\right) / \sqrt{2}
$$

The last term in the gravitino equation (2.7) using (2.1) is

$$
\frac{i}{480} \mathcal{F}_{P Q R S T} \Gamma^{P Q R S T} \gamma_{M}=\frac{i}{4} f(u)\left(P_{1}+P_{2}\right) \gamma_{-} \gamma_{M},
$$

where

$$
P_{1}=\gamma_{1} \gamma_{2} \gamma_{3} \gamma_{4}, \quad P_{2}=\gamma_{5} \gamma_{6} \gamma_{7} \gamma_{8}
$$

are products of Gamma matrices acting as projection operators in the four-dimensional subspaces defined by the five-form ansatz in (2.1). The obey

$$
\begin{aligned}
P_{1}^{2} & =P_{2}^{2}=1, \quad\left[P_{1}, P_{2}\right]=\left[P_{1,2}, \gamma_{ \pm}\right]=0, \\
\left\{P_{1}, \gamma_{i}\right\} & =\left[P_{1}, \gamma_{i+4}\right]=0, \quad i=1,2,3,4, \\
\left\{P_{2}, \gamma_{i}\right\} & =\left[P_{2}, \gamma_{i-4}\right]=0, \quad i=5,6,7,8 .
\end{aligned}
$$

Combined with (2.8), we find (2.7) in components

$$
\begin{aligned}
\partial_{-} \epsilon & =0 \\
\partial_{i} \epsilon & =-\frac{i}{4} f(u) \Omega^{i} \epsilon, \\
\partial_{+} \epsilon & =-\frac{1}{2} F_{i j} x^{j} \gamma_{-} \gamma_{i} \epsilon-\frac{i}{4} f(u)\left(P_{1}+P_{2}\right) \gamma_{-} \gamma_{+} \epsilon,
\end{aligned}
$$

where

$$
\Omega_{i}=\left(P_{1}+P_{2}\right) \gamma_{-} \gamma_{i}
$$

obeying

$$
\left[P_{1}+P_{2}, \Omega_{i}\right]=2\left(1+P_{1} P_{2}\right) \gamma_{-} \gamma_{i} .
$$

The first condition in (2.13) states that $\epsilon$ is a function of $x^{i}$ and $x^{+}=u$ only. The second condition can be solved by using the fact that $\Omega^{i} \Omega^{j}=0$, which implies that $\partial_{i} \partial_{j} \epsilon=0$, i.e. $\epsilon$ is linear in the $x^{i}$. The solution is

$$
\epsilon=\left(1-\frac{i}{4} f(u) x^{j} \Omega^{j}\right) \chi(u),
$$

which we insert in the third condition in (2.13) resulting in a linear equation in $x^{i}$. Hence we obtain two condition that read

$$
\left(\partial_{u}+\frac{i}{4} f(u)\left(P_{1}+P_{2}\right) \gamma_{-} \gamma_{+}\right) \chi=0
$$

and

$$
x^{i}\left(i f^{\prime}(u)\left(P_{1}+P_{2}\right) \gamma_{i}-2 F_{i j} \gamma_{j}-2 f^{2} \gamma_{i}\right) \gamma_{-} \chi=0 .
$$

In the various manipulations we have used repeatedly that $\gamma_{ \pm}^{2}=0$ and that

$$
\left(1 \mp P_{1} P_{2}\right) \chi=\gamma_{ \pm} \gamma_{\mp} \chi,
$$

which is a consequence of the chirality condition $\gamma^{11} \epsilon=\epsilon$ and the fact that $\gamma^{11}$ anticommutes with all Gamma matrices. 
For generic $f$ and $F_{i j}$ this equation has 16 solutions given by $\gamma^{+} \chi=\gamma_{-} \chi=0$ and the explicit $u$-dependence is easily found by integrating (2.17). The maximal supersymmetric solution with 32 supercharges corresponds to constant $f$ and $F_{i j}$ related by $F_{i j}=-f^{2} \delta_{i j}$, $i=1, \ldots, 8$. In cases where the function $f(u)$ is not a constant there exist in general no additional real supersymmetric solutions. To see this, note that for spinors obeying $\gamma-\chi=0$ we have, due to (2.19), one of two possibilities: $P_{1} \chi=P_{2} \chi= \pm \chi$. Therefore, any additional supersymmetries must meet one of the two remaining possibilities: $P_{1} \chi=-P_{2} \chi= \pm \chi$. However, using the fact that $\gamma_{i}$ commutes with one of the projectors among $P_{1}, P_{2}$ and anti-commutes with the other (2.12), we see that the eq. (2.18) will give rise to complex $F_{i j}$ and, hence, to complex metrics which are physically unacceptable.

\section{Rotating D3-branes and Penrose limits}

In this section we construct Penrose limits of the supergravity solutions of rotating D3branes [22, 23]. The most general solution is characterized by five constants: the number of D3-branes, $N$, three rotation parameters, $r_{1,2,3}$, and the near-extremality parameter, $\mu$, which is related to the Hawking temperature of the black brane solution. For general $r_{i}$ the isometry of the transverse space, which is related to the $\mathcal{R}$-symmetry in the dual field theory, is reduced $\mathrm{SO}(6) \rightarrow \mathrm{SO}(2) \times \mathrm{SO}(2) \times \mathrm{SO}(2)$. We will not consider the most general case, but we will restrict our attention to backgrounds with only one non-vanishing rotation parameter: $r_{1}=r_{0}, r_{2}=r_{3}=0$. Then the symmetry of the solution is $I \mathrm{SO}(3,1) \times \mathrm{SO}(4) \times$ $\mathrm{SO}(2)$. The explicit form of the metric and RR five-form field strength of the supergravity solution, in the field theory limit, is [23]

$$
\begin{aligned}
d s^{2}= & H^{-1 / 2}\left(-f d t^{2}+d x_{1}^{2}+d x_{2}^{2}+d x_{3}^{2}\right) \\
& +H^{1 / 2}\left(\frac{d r^{2}}{f_{1}}+\left(r^{2}+r_{0}^{2} \cos ^{2} \theta\right) d \theta^{2}+\left(r^{2}+r_{0}^{2}\right) \sin ^{2} \theta d \phi^{2}+r^{2} \cos ^{2} \theta d \Omega_{3}^{2}-\frac{2 \mu^{2} r_{0}}{R^{2}} \sin ^{2} \theta d t d \phi\right), \\
\mathcal{F}_{5}= & d C_{4}+* d C_{4} \quad \text { with } \\
C_{4}= & \left(H^{-1} d t+r_{0} \mu^{2} / R^{2} \sin ^{2} \theta d \phi\right) \wedge d x^{1} \wedge d x^{2} \wedge d x^{3}
\end{aligned}
$$

and

$$
\begin{aligned}
H & =\frac{R^{4}}{r^{2}\left(r^{2}+r_{0}^{2} \cos ^{2} \theta\right)} \\
f & =1-\frac{\mu^{4}}{r^{2}\left(r^{2}+r_{0}^{2} \cos ^{2} \theta\right)} \\
f_{1} & =\frac{r^{4}+r_{0}^{2} r^{2}-\mu^{4}}{r^{2}\left(r^{2}+r_{0}^{2} \cos ^{2} \theta\right)}
\end{aligned}
$$

where $R^{4}=4 \pi g_{s} N$ and $r_{0}$ is the angular momentum parameter. Note that we set $\alpha^{\prime}=1$ throughout the paper. The location of the horizon is given by the positive root of the equation $r^{4}+r_{0}^{2} r^{2}-\mu^{4}=0$

$$
r_{H}^{2}=\frac{1}{2}\left(\sqrt{r_{0}^{4}+4 \mu^{4}}-r_{0}^{2}\right)
$$


and the Hawking temperature associated with (3.1) is

$$
T_{H}=\frac{r_{H}}{2 \pi R^{2} \mu^{2}} \sqrt{r_{0}^{4}+4 \mu^{4}}
$$

The background (3.1) has been used in various studies within the AdS/CFT correspondence in 23, 24, 25].

In order to take a Penrose limit we have to choose a null geodesic on this space. This will in general involve the time directions $t$, the radius $r$ and some directions on the transverse $S^{5}$. In contrast to the maximally supersymmetric case the $S^{5}$ is now squashed, hence the isometry is reduced, and there are several inequivalent choices for the geodesics. To make life a bit easier we only consider geodesics with constant $\theta$ and this leaves two consistent possibilities: $\theta=\pi / 2$ or $\theta=0$.

\subsection{Null geodesics}

The null geodesics that are relevant for taking Penrose limits involve the directions $t, r$ and a particular angular direction which we denote for the moment by $\alpha$. All other angular directions and the flat spatial coordinates along the brane $x_{1,2,3}$ are taken to be constant. After a convenient rescaling $x^{\mu} \rightarrow R^{2} x^{\mu}$, we find a three-dimensional effective metric

$$
d s_{3}^{2} / R^{2}=-G_{t} d t^{2}+G_{r} d r^{2}+G_{\alpha} d \alpha^{2}-2 G_{t \alpha} d t d \alpha,
$$

which contains an off-diagonal term due to the angular momentum.

We are looking for null geodesics parametrized by $r, t, \alpha$ as a function of the proper time $\tau$. Conservation of energy and angular momentum implies that

$$
G_{t} \dot{t}+G_{t \alpha} \dot{\alpha}=E \equiv 1, \quad G_{\alpha} \dot{\alpha}-G_{t \alpha} \dot{t}=J,
$$

with solution

$$
\dot{t}=\frac{G_{\alpha}-J G_{t \alpha}}{G_{t \alpha}^{2}+G_{t} G_{\alpha}}, \quad \dot{\alpha}=\frac{G_{t \alpha}+J G_{t}}{G_{t \alpha}^{2}+G_{t} G_{\alpha}} .
$$

These expressions can now be fed back into the effective line element (3.5) and requiring the geodesic to be null yields a differential equation for $r$

$$
\dot{r}^{2}=\frac{G_{\alpha}-2 J G_{t \alpha}-J^{2} G_{t}}{G_{r}\left(G_{t \alpha}^{2}+G_{t} G_{\alpha}\right)} .
$$

For completeness we present here also the general form of a particular change of coordinates that is important for taking the Penrose limit (see also [26])

$$
\begin{aligned}
d r & =\sqrt{\frac{G_{\alpha}-2 J G_{t \alpha}-J^{2} G_{t}}{G_{r}\left(G_{t \alpha}^{2}+G_{t} G_{\alpha}\right)}} d u, \\
d t & =\frac{G_{\alpha}-J G_{t \alpha}}{G_{t \alpha}^{2}+G_{t} G_{\alpha}} d u-\frac{1}{R^{2}} d v+\frac{J}{R} d x, \\
d \alpha & =\frac{G_{t \alpha}+J G_{t}}{G_{t \alpha}^{2}+G_{t} G_{\alpha}} d u+\frac{1}{R} d x .
\end{aligned}
$$

Comparing with (3.8) we see that $u$ plays the role of the proper time in the null geodesic. 


\subsection{Geodesics at $\theta=\pi / 2$}

When $\theta=\pi / 2$ we see that the $S^{3}$ part in (3.1) vanishes and the non-trivial angular direction is $\alpha \equiv \phi$. In this case the metric elements of the three-dimensional effective metric (3.5) are

$$
G_{t}=r^{2}-\frac{\mu^{4}}{r^{2}}, \quad G_{r}=\frac{r^{2}}{r^{4}+r_{0}^{2} r^{2}-\mu^{4}}, \quad G_{\phi}=1+\frac{r_{0}^{2}}{r^{2}}, \quad G_{t \phi}=\frac{\mu^{2} r_{0}}{r^{2}}
$$

The general (real) solution of (3.8) turns out to be

$$
r^{2}(u)=\frac{1}{2 J^{2}}(1-a \cos 2 J u), \quad a \equiv \sqrt{1+4\left(r_{0}-J \mu^{2}\right)^{2} J^{2}} .
$$

For the Penrose limit we perform the change of variables (3.9), set

$$
\theta=\frac{\pi}{2}-\frac{z}{R}, \quad x_{1,2,3} \rightarrow x_{1,2,3} / R,
$$

and define $d z^{2}+z^{2} d \Omega_{3}^{2}=d \vec{x}_{4}^{2}$, so that $z^{2}=\vec{x}_{4}^{2}$. Furthermore, we combine the spacelike brane directions into the three-vector $\vec{x}_{3}$. The Penrose limit of (3.1) is then obtained by employing all these coordinate transformations and taking the limit $R \rightarrow \infty$. The resulting metric is

$$
d s^{2}=2 d u d v+d \vec{x}_{4}^{2}+A_{3}(u) d \vec{x}_{3}^{2}+A_{x}(u) d x^{2}-J^{2} \vec{x}_{4}^{2} d u^{2},
$$

where the various functions are

$$
\begin{aligned}
& A_{x}=1+r_{0}^{2} / r^{2}-J^{2} r^{2}=\frac{1}{2} \frac{a^{2} \sin ^{2} 2 J u}{1-a \cos 2 J u}, \\
& A_{3}=r^{2}=\frac{1}{2 J^{2}}(1-a \cos 2 J u)
\end{aligned}
$$

Furthermore, it will be useful to present the metric in a different form using Brinkman coordinates. ${ }^{2}$ We find the metric

$$
d s^{2}=2 d u d v+d x^{2}+d \vec{x}_{3}^{2}+d \vec{x}_{4}^{2}+\left(F_{x} x^{2}+F_{3} \vec{x}_{3}^{2}+F_{4} \vec{x}_{4}^{2}\right) d u^{2},
$$

where

$$
\begin{aligned}
& F_{x}=-J^{2}\left(1-3 \frac{a^{2}-1}{(1-a \cos 2 J u)^{2}}\right), \\
& F_{3}=-J^{2}\left(1+\frac{a^{2}-1}{(1-a \cos 2 J u)^{2}}\right), \\
& F_{4}=-J^{2}
\end{aligned}
$$

Note that the metric depends explicitly on light-cone time $u$. From the discussion around (2.1) and (2.6) and the form (3.13), we understand that this background has an

\footnotetext{
${ }^{2}$ For a metric of the form $d s^{2}=2 d u d v+A d x^{2}$ this means that $u \rightarrow u, v \rightarrow v+\frac{A^{\prime}}{4 A} x^{2}, x \rightarrow x / \sqrt{A}$, which then gives the metric $d s^{2}=2 d u d v+d x^{2}+F x^{2} d u^{2}$, where $F=\frac{1}{4} A^{\prime 2} / A^{2}+\frac{1}{2}\left(A^{\prime} / A\right)^{\prime}$. In the case of several transverse directions this transformation is trivially iterated. If there is a $d u^{2}$ component in the original metric then this is absorbed in the definition of $F$ above, making sure that we also rescale $x$ as above.
} 
$\mathrm{SO}(3) \times \mathrm{SO}(4) \times \mathrm{U}(1)$ symmetry. The $\mathrm{U}(1)$ factor is not manifest in the Brinkman coordinates, in contrast to the Rosen-like coordinates (B.13). ${ }^{3}$ We also find that the Penrose limit taken for the five-form in (3.1) gives an expression of the form (2.1), namely

$$
\mathcal{F}_{5}=J d u \wedge\left(1+*_{8}\right)\left(d x \wedge d x_{1} \wedge d x_{2} \wedge d x_{3}\right) .
$$

It is quite remarkable that the five-form in the particular PP-wave limit that we have taken does not depend at all on the non-extremality parameter $\mu$ and on the vev parameter $r_{0}$, but, instead, it retains the form it has for the maximally supersymmetric PP-wave solution. This has, as we will see, the important consequence that the fermionic spectrum in the Green-Schwarz action can be immediately determined. In addition, in this case, the temperature effects are washed out when the Penrose limit is taken. This is also seen by the fact that, in the metric, $\mu$ and $r_{0}$ always appear combined into the constant $a$ defined in (3.11). Therefore, in order to recover the maximally supersymmetric PP-wave solution it is not required that both $\mu$ and $r_{0}$ be set to zero, but simply that $r_{0}=J \mu^{2}$.

\subsection{Geodesics at $\theta=\pi / 2$ and zero temperature}

It is also of interest to consider the extremal limit $\mu \rightarrow 0$. In this case the background (3.1) becomes

$$
\begin{aligned}
d s^{2}= & \frac{r\left(r^{2}+r_{0}^{2} \cos ^{2} \theta\right)^{1 / 2}}{R^{2}} \eta_{\mu \nu} d x^{\mu} d x^{\nu}+\frac{R^{2}}{r\left(r^{2}+r_{0}^{2} \cos ^{2} \theta\right)^{1 / 2}} \times \\
& \times\left(\left(r^{2}+r_{0}^{2} \cos ^{2} \theta\right)\left(\frac{d r^{2}}{r^{2}+r_{0}^{2}}+d \theta^{2}\right)+\left(r^{2}+r_{0}^{2}\right) \sin ^{2} \theta d \phi^{2}+r^{2} \cos ^{2} \theta d \Omega_{3}^{2}\right),
\end{aligned}
$$

which describes a uniform distribution of D3-branes over a disk of radius $r_{0}$, or after we send $r_{0} \rightarrow-i r_{0}$, a uniform distribution of D3-branes over a three-sphere. Both backgrounds preserve sixteen supercharges and in the AdS/CFT context they describe particular points in the Coulomb branch of $\mathcal{N}=4 \mathrm{SYM}$ in the regime of large t'Hooft coupling. Various related studies have been performed in [27, 25, 28].

Without extra work we can use the results of the previous section directly and apply the limit $\mu \rightarrow 0$. The PP-wave metric in Brinkman coordinates have the same form as in (3.13) - 3.16), the only difference being that the definition for $a$ in (3.11) has to be replaced by

$$
a=\sqrt{1+4 r_{0}^{2} J^{2}} .
$$

So far we have assumed that the parameter $r_{0}^{2}$ is positive, which corresponds to a distribution of branes on the two-plane (corresponding to $\theta=\pi / 2$ in our parametrization). Hence the geodesic that we have followed $(\theta=\pi / 2)$ probes the region near the branedistribution. Notice also that the maximum value for $r^{2}$ is $(1+a) /\left(2 J^{2}\right)$ which for small $r_{0}$ becomes $1 / J^{2}$ whereas for large $r_{0}$ it becomes $r_{0} J$.

If we want to consider the sphere distribution we have to take $r_{0}^{2} \rightarrow-r_{0}^{2}$ which implies $a=\sqrt{1-4 r_{0}^{2} J^{2}}$. Hence, only for $r_{0} J<1 / 2$ the constant $a$ is real and we can keep

\footnotetext{
${ }^{3}$ In general, in the notation of footnote 2 , the $\mathrm{U}(1)$ symmetry acts non-trivially as: $\delta x=\sqrt{A} \epsilon$ and $\delta v=-\frac{1}{2} x A^{\prime} / \sqrt{A} \epsilon$, where $\epsilon$ is an infinitesimal constant parameter.
} 
our previous formulas for the metrics. However, for $r_{0} J>1 / 2$ the constant $a$ becomes imaginary and the expressions become unphysical. In particular, there are no real solutions to the geodesic equation (eq. (3.8) with $\mu=0$ ) and the PP-wave metric and the RR fiveform flux becomes imaginary.

\subsection{Geodesics at $\theta=0$}

The procedure is very similar to the $\theta=\pi / 2$ case, so we will be brief. In (3.1) we replace $d \Omega_{3}^{2}$ by

$$
d \Omega_{3}^{2}=d \omega^{2}+\cos ^{2} \omega d \psi^{2}+\sin ^{2} \omega d \tilde{\psi}^{2} .
$$

We take the geodesic located at $\omega=\tilde{\psi}=0$ and the non-trivial angular coordinate along the geodesic is $\alpha=\psi$. The non-zero coefficients of the effective three-dimensional metric (3.5) are

$$
G_{t}=\frac{r^{2}\left(r^{2}+r_{0}^{2}\right)-\mu^{4}}{r \sqrt{r^{2}+r_{0}^{2}}}, \quad G_{r}=1 / G_{t}, \quad G_{\psi}=1 / \sqrt{1+\frac{r_{0}^{2}}{r^{2}}} .
$$

The null geodesic equations (3.8) can easily be solved

$$
r^{2}(u)=\frac{1}{2 J^{2}}\left(1-J^{2} r_{0}^{2}-b^{2} \cos 2 J u\right)
$$

with $b^{2}=\sqrt{\left(1-r_{0}^{2} J^{2}\right)^{2}+4 J^{4} \mu^{4}}$.

As before, we make the change of variables (3.9) and let

$$
\theta=\frac{z}{R}, \quad \omega=\frac{\tilde{z}}{R}, \quad x_{1,2,3} \rightarrow x_{1,2,3} / R .
$$

In addition, we define $d z^{2}+z^{2} d \phi^{2}=d \vec{x}_{2}^{2}$, so that $z^{2}=\vec{x}_{2}^{2}$, and $d \tilde{z}^{2}+\tilde{z}^{2} d \tilde{\psi}^{2}=d \overrightarrow{\tilde{x}}_{2}^{2}$, so that $\tilde{z}^{2}=\overrightarrow{\vec{x}}_{2}^{2}$. We also assemble the three spacelike brane directions into the three-vector $\vec{x}_{3}$. In the Penrose limit $R \rightarrow \infty$ the metric takes the form

$$
d s^{2}=2 d u d v+A_{2}(u) d \vec{x}_{2}^{2}+\tilde{A}_{2}(u) d \overrightarrow{\tilde{x}}_{2}^{2}+A_{3}(u) d \vec{x}_{3}^{2}+A_{x}(u) d x^{2}-C(u, z, \tilde{z}) d u^{2},
$$

where the numerous functions are given by:

$$
\begin{aligned}
A_{x} & =\frac{1}{\Delta}-J^{2} r^{2} \Delta+\frac{J^{2} \mu^{4}}{r^{2} \Delta}, \\
A_{3} & =r^{2} \Delta \\
A_{2} & =1 / \tilde{A}_{2}=\Delta, \\
C & =J^{2}\left(\frac{\vec{x}^{2}}{\Delta}+\overrightarrow{\tilde{x}}^{2} \Delta\right)-\frac{\mu^{4} r_{0}^{2} \vec{x}^{2}}{\Delta\left(r^{4}+r^{2} r_{0}^{2}-\mu^{4}\right)^{2}}, \\
\Delta & =\sqrt{1+r_{0}^{2} / r^{2}} .
\end{aligned}
$$

We refrain from presenting the metric in Brinkman coordinates, since the formulas turn out to be quite cumbersome and not very illuminating. However, we note that, unlike the PP-wave corresponding to the geodesic at $\theta=\pi / 2$, in this case temperature effects parametrized by the constant $\mu$ remain distinct from those parametrized by the rotational parameter $r_{0}$, i.e., $\mu$ and $r_{0}$ do not combine into a single constant, as before. 


\subsection{Geodesics at $\theta=0$ and zero temperature}

The extremal limit is obtained by setting $\mu \rightarrow 0$ in all expressions of the previous subsection which in particular implies $b \equiv \sqrt{1-r_{0}^{2} J^{2}}$. Now, transforming the PP-wave (3.24) and (3.25) to Brinkman coordinates we find in the extremal limit

$$
d s^{2}=2 d u d v+d x^{2}+d \vec{x}_{3}^{2}+d \vec{x}_{2}^{2}+d \overrightarrow{\tilde{x}}_{2}^{2}+\left(F_{x} x^{2}+F_{3} \vec{x}_{3}^{2}+F_{2} \vec{x}_{2}^{2}+\tilde{F}_{2} \overrightarrow{\tilde{x}}_{2}^{2}\right) d u^{2},
$$

where

$$
\begin{aligned}
& F_{x}=-J^{2}\left(1-\frac{5}{4} \frac{b^{2}-1}{\left(1-b^{2} \cos ^{2} J u\right)^{2}}-\frac{1}{4} \frac{b^{2}-1}{\sin ^{2} J u\left(1-b^{2} \cos ^{2} J u\right)}\right), \\
& F_{3}=-J^{2}\left[1+\frac{b^{2}-1}{4\left(1-b^{2} \cos ^{2} J u\right)^{2}}\left(\frac{b^{2}-1}{\sin ^{2} J u}-b^{2}+3\right)\right], \\
& F_{2}=-J^{2}\left(\frac{b^{2} \sin ^{2} J u}{1-b^{2} \cos ^{2} J u}-\frac{\left(b^{2}-1\right)\left(4 b^{2} \cos ^{4} J u-2-\left(b^{2}+1\right) \cos ^{2} J u\right)}{4\left(1-b^{2} \cos ^{2} J u\right)^{2} \sin ^{2} J u}\right), \\
& \tilde{F}_{2}=-J^{2}\left(\frac{1-b^{2} \cos ^{2} J u}{b^{2} \sin ^{2} J u}+\frac{\left(b^{2}-1\right)\left(4 b^{2} \cos ^{4} J u-2+\left(1-3 b^{2}\right) \cos ^{2} J u\right)}{4\left(1-b^{2} \cos ^{2} J u\right)^{2} \sin ^{2} J u}\right) .
\end{aligned}
$$

We see that for $r_{0} J \leq 1$ the constant $b$ is real and all expressions make sense, but when $r_{0} J>1$ then the background becomes complex and is unphysical. On the other hand if go to the sphere distribution (see comments after eq. 3.18) via analytic continuation $r_{0}^{2} \rightarrow-r_{0}^{2}$. All the expressions we remain valid, with $b=\sqrt{1+J^{2} r_{0}^{2}}$, without restrictions on $r_{0} J$.

For the RR five-form field strength in (3.1), in the PP-limit, we find that

$$
\begin{aligned}
\mathcal{F}_{5} & =f(u) d u \wedge\left(1+*_{8}\right)\left(d x \wedge d x_{1} \wedge d x_{2} \wedge d x_{3}\right) \\
& =\frac{J}{2 b} \frac{1-b^{2} \cos 2 J u}{\sin J u \sqrt{1-b^{2} \cos ^{2} J u}} d u \wedge\left(1+*_{8}\right)\left(d x \wedge d x_{1} \wedge d x_{2} \wedge d x_{3}\right),
\end{aligned}
$$

which unlike the $\theta=\pi / 2$ case depends explicitly on $u$. This can be read off from the equation of motion (2.6) which gives the relation

$$
8 f^{2}(u)=-\left(F_{x}+3 F_{3}+2 F_{2}+2 \tilde{F}_{2}\right) .
$$

For the case of the sphere distribution, i.e. $r_{0}^{2}>0$, we may consider the limit $b \rightarrow \infty$. The naive limit leads to a well defined expression for the metric (see (3.27)), but $\mathcal{F}_{5}$ becomes imaginary. The reason is that $\cos ^{-1}(1 / b) \leq J u \leq \pi / 2$, which means that $J u$ is pushed towards the value $J u=\pi / 2$. Therefore, we will consider the following correlated limit

$$
J u \rightarrow \pi / 2-u / b, \quad v \rightarrow-b J v,
$$

followed by the limit $b \rightarrow \infty$. Then the metric and five-form are well behaved and the metric maintains its original form, but with

$$
\begin{array}{ll}
F_{x}=\frac{6-u^{2}}{4\left(1-u^{2}\right)^{2}}, & F_{3}=-\frac{u^{2}+2}{4\left(1-u^{2}\right)^{2}}, \\
F_{2}=\frac{3\left(u^{2}-2\right)}{4\left(1-u^{2}\right)^{2}}, & \tilde{F}_{2}=\frac{3 u^{2}+2}{4\left(1-u^{2}\right)^{2}},
\end{array}
$$


where the variable $0 \leq u \leq 1$. The five-form field strength in this limit becomes

$$
\begin{aligned}
\mathcal{F}_{5} & =f(u) d u \wedge\left(1+*_{8}\right)\left(d x \wedge d x_{1} \wedge d x_{2} \wedge d x_{3}\right) \\
& =-\frac{1}{2} \frac{1}{\sqrt{1-u^{2}}} d u \wedge\left(1+*_{8}\right)\left(d x \wedge d x_{1} \wedge d x_{2} \wedge d x_{3}\right) .
\end{aligned}
$$

It can be checked directly that (3.29) is satisfied.

\section{The perturbative string spectrum}

In this section we study the perturbative string spectrum by studying the corresponding two-dimensional Green-Schwarz action. The only background fields that couple to the bosonic string modes are the metric and the NS-NS two-form, where the latter is zero for our backgrounds. Hence, the bosonic part of the Green-Schwarz action is just the Polyakov action:

$$
S_{B}=\frac{1}{4 \pi} \int d \tau d \sigma g_{\mu \nu} \partial_{a} x^{\mu} \partial^{a} x^{\nu}
$$

with $\sigma \sim \sigma+2 \pi$.

For PP-waves the most natural gauge choice is the light-cone gauge

$$
u=P_{+} \tau
$$

where, without loss of generality, a possible additive constant has been set to zero. If the metric is of the form (2.1) the fluctuations of the eight transverse modes are governed by Schrödinger equations (see, for instance, [29])

$$
-\frac{d^{2} x_{n}^{i}}{d \tau^{2}}+P_{+}^{2} F_{i}\left(P_{+} \tau\right) x_{n}^{i}=n^{2} x_{n}^{i}, \quad(\text { no sum over i) }
$$

where we have performed a Fourier transform on the $x^{i}$ in the world-sheet direction $\sigma$. Note that this formula is only valid if $F_{i j}$ is diagonal which is the case for our backgrounds.

The Schrödinger equations for the scalar modes with potentials given by the metric functions $F_{i}$ in the previous section are in general hard to solve analytically. However, in many cases it is possible to make connection with supersymmetric quantum mechanics (SQM) 30. ${ }^{4}$ This is a consequence of the relation between the $F_{i}$ and $A_{i}$ functions appearing in the metrics in Brinkman and Rosen coordinates, respectively. The crucial formula was given in footnote 2 and is nothing but the expression of the Schrödinger potentials $F_{i}$ in terms of prepotentials $W_{i}{ }^{5}$

$$
F_{i}=W_{i}^{2}-W_{i}^{\prime}, \quad W_{i}=-\frac{1}{2} A_{i}^{\prime} / A_{i}
$$

with ground state wave function

$$
\Psi_{0}=\sqrt{A_{i}} .
$$

\footnotetext{
${ }^{4}$ For a comprehensive review on this subject see [31].

${ }^{5}$ This works straightforwardly only if the metric in Rosen-like coordinates has no $d u^{2}$ term for the corresponding coordinate. For instance, this is the case for the coordinates $x$ and $\vec{x}_{3}$ in (3.13), but not for $\vec{x}_{2}$ and $\overrightarrow{\tilde{x}}_{2}$ in (3.24). Nevertheless, this can be done for all coordinates with some extra work.
} 
Spectra of SQM are positive definite and a zero-mode exists provided that the norm $\Psi_{0}$ is finite i.e. $\left|\int A_{i}\right|<\infty$. There exist large classes of exactly solvable SQM problems [31] and in cases where analytic methods fail, the WKB approximation for SQM does usually better than for generic quantum mechanical potentials. Some exactly solvable cases will be discussed later in this section. We also note that (4.5) represents a solution to the null geodesic equations corresponding to the metric in (2.1).

In order to find the fermion spectrum we need to extract the part of the Green-Schwarz action that is quadratic in fermions. The relevant formulas have been presented in several papers in the literature, e.g. [3]. The generalization of the Dirac operator that appears in the fermion kinetic term is just the differential operator that appears in the gravitino variation of type-IIB supergravity. Hence, the fermionic part of the GS action takes the form

$$
S_{F}=\int d \tau d \sigma i\left(\eta^{a b} \delta_{I J}-\epsilon^{a b} \rho_{3 I J}\right) \partial_{a} x^{\mu} \bar{\theta}^{I} \Gamma_{\mu} \mathcal{D}_{b} \theta^{J}
$$

where $\eta^{00}=-\eta^{11}=-1, \epsilon^{01}=1$ and $\rho_{3}=\operatorname{diag}(1,-1)$. The derivation $\mathcal{D}_{b}$ is the pull-back of the differential operator that appears in the gravitino variation:

$$
\mathcal{D}_{a} \equiv \partial_{a}+\partial_{a} x^{\mu}\left(\frac{1}{4} \omega_{\mu A B} \Gamma^{A B}+\mathcal{F}_{A B C D E} \Gamma^{A B C D E} \rho_{0} \Gamma_{\mu}\right)
$$

with $\rho_{0}=i \sigma_{2}$.

For our type of backgrounds (2.1) this simplifies to

$$
\mathcal{D}_{a}\left(\begin{array}{c}
\theta_{1} \\
\theta_{2}
\end{array}\right)=\partial_{a}\left(\begin{array}{c}
\theta_{1} \\
\theta_{2}
\end{array}\right)+\partial_{a} x^{+}\left[\frac{1}{4} \omega_{+M N} \gamma^{M N}\left(\begin{array}{c}
\theta_{1} \\
\theta_{2}
\end{array}\right)+\frac{f(u)}{2} \gamma_{+}\left(P_{1}+P_{2}\right)\left(\begin{array}{c}
\theta_{2} \\
-\theta_{1}
\end{array}\right)\right] .
$$

Using this and the light-cone gauge the fermion equations become

$$
\gamma_{+}\left(\begin{array}{c}
\left(\partial_{\tau}+\partial_{\sigma}\right) \theta_{1} \\
\left(\partial_{\tau}-\partial_{\sigma}\right) \theta_{2}
\end{array}\right)=P_{+} \frac{f\left(P_{+} \tau\right)}{2} \gamma_{+}\left(P_{1}+P_{2}\right)\left(\begin{array}{c}
\theta_{2} \\
-\theta_{1}
\end{array}\right)
$$

We expand the fermions in Fourier modes in the $\sigma$ direction. Note also that in addition to the chirality conditions $\theta_{i}=\gamma_{11} \theta_{i}$ the light-cone gauge implies $\gamma_{-} \theta_{i}=0$. In the conventions of [2] that means that out of the 32 components of the $\theta_{i}$ only the first eight components are non-zero and we have $P_{1} \theta_{i}=P_{2} \theta_{i}$, where $P_{1}$ and $P_{2}$ are the projectors defined in (2.11). This also follows immediately from the fact that a condition similar to (2.19) holds for spinors $\theta_{i}$ as well. Consequently we can ignore the $\gamma_{+}$factors in (4.9) and arrange the remaining fermionic degrees of freedom in two 8-component spinors $\tilde{\theta}_{1,2}$. In the last step we reshuffle the total of 16 spinor components of $\tilde{\theta}_{1,2}$ and redistribute them in two 8-component spinors $\psi_{1,2}$ such that the differential equation (4.9) is of the form

$$
\left(\begin{array}{c}
\partial_{\tau} \psi_{1} \\
\partial_{\tau} \psi_{2}
\end{array}\right)=\left(\begin{array}{cc}
-i n & -P_{+} f \\
P_{+} f & i n
\end{array}\right)\left(\begin{array}{c}
\psi_{1} \\
\psi_{2}
\end{array}\right)
$$

If $f$ is constant this equation can easily be solved and gives harmonic oscillators with frequencies

$$
\omega= \pm \sqrt{n^{2}+P_{+}^{2} f^{2}}
$$


with each sign having multiplicity eight. If $f$ depends non-trivially on light-cone time we can eliminate one of the fermions in terms of the other at the cost of introducing a second order differential equation:

$$
\psi_{1}^{\prime \prime}-P_{+} f^{\prime} / f \psi_{1}^{\prime}+\left(n^{2}+P_{+}^{2} f^{2}-i n P_{+} f^{\prime} / f\right) \psi_{1}=0,
$$

where the prime denotes the derivative with respect to the argument.

\subsection{Spectra en détail}

We start with the $\mathrm{PP}$-wave corresponding to the geodesic with $\theta=\pi / 2$. The spectrum corresponding to the $\vec{x}_{4}$ directions is like in the maximally supersymmetric case [2]. The oscillator frequencies are

$$
\omega_{4}=\sqrt{n^{2}+J^{2} P_{+}^{2}}, \quad n=1,2,3, \ldots
$$

For the other directions let $z=2 J P_{+} \tau$. Then, using (3.16) and (4.3), we find the two Schrödinger equations (we will collectively denote by $\Psi(z)$ the corresponding $x_{n}^{i}$ 's)

$$
-\frac{d^{2} \Psi}{d z^{2}}-\frac{1}{4}\left(1+\frac{a^{2}-1}{(1-a \cos z)^{2}}\right) \Psi=\frac{n^{2}}{4 J^{2} P_{+}^{2}} \Psi, \quad n=0,1, \ldots
$$

and

$$
-\frac{d^{2} \Psi}{d z^{2}}-\frac{1}{4}\left(1-3 \frac{a^{2}-1}{(1-a \cos z)^{2}}\right) \Psi=\frac{n^{2}}{4 J^{2} P_{+}^{2}} \Psi, \quad n=0,1, \ldots
$$

For general $a$ the two potentials are not supersymmetric partners.

For $a>1$, i.e. the case of a disk, let us define the constant angle $z_{0}=\cos ^{-1} 1 / a$ which takes values in the interval $z_{0} \in\left[0, \frac{\pi}{2}\right]$. Then the allowed coordinate ranges are, either $z_{0} \leq z \leq \pi$ or $-z_{0} \leq z \leq z_{0}$. Near $z= \pm z_{0}$ the potentials behave as $-\frac{1}{4} \frac{1}{\left(z-z_{0}\right)^{2}}$ and $\frac{3}{4} \frac{1}{\left(z-z_{0}\right)^{2}}$, respectively, which means that there is no unitarity problem in these cases, as expected from SQM. For $z=\pi$ they go to a constant. When $a<1$, i.e. the case of a sphere, the potentials are nowhere singular.

Now let us consider the limit $a \rightarrow \infty$ for which the first coordinate range becomes $\pi / 2 \leq z \leq \pi$. Shifting the $z$ variable as $z=x+\pi / 2$ we obtain the range $0 \leq x \leq \pi / 2$. Then the two potentials are of the Pöschl-Teller type-I, the normalizable solutions are given in terms of Jacobi polynomials and the spectra are quantized with boundary conditions $\Psi(0)=\Psi(\pi / 2)=0$. For (4.14) (with $a \rightarrow \infty)$ we find

$$
\Psi_{m}=\cos x(\sin x)^{1 / 2} P_{m}^{\left(0, \frac{1}{2}\right)}(\cos 2 x), \quad\left(\frac{n}{4 J P_{+}}\right)^{2}=(m+1)\left(m+\frac{1}{2}\right),
$$

and for (4.15) (with $a \rightarrow \infty$ )

$$
\Psi_{m}=\cos x(\sin x)^{3 / 2} P_{m}^{\left(1, \frac{1}{2}\right)}(\cos 2 x), \quad\left(\frac{n}{4 J P_{+}}\right)^{2}=(m+1)\left(m+\frac{3}{2}\right)
$$

with $m=0,1,2, \ldots$, for both cases. We note that for $a \rightarrow \infty$ the two potentials in (4.14) and (4.15) are supersymmetric partners. However, the corresponding spectra as 
given above are different. The reason is that we have imposed the boundary condition of vanishing wavefunctions at the end points of the interval $x \in[0, \pi / 2]$. However, it can be shown that these boundary conditions are not obeyed by both sets of wavefunctions if they are simply related via the usual rules of SQM. We see that the constant $P_{+} J$ needs to be quantized in order for the wavefunctions that obey the appropriate boundary conditions to be normalizable. For generic values of $P_{+} J$ we should take the wavefunction to be trivial, i.e. $\Psi=0$. Hence, for generic values of $P_{+} J$ we have only the spectra corresponding to $\vec{x}_{4}$ with oscillator frequencies given by (4.13). When $P_{+} J$ is quantized according to (4.16) or (4.17) we have in addition the excitations of the corresponding coordinates.

For the second coordinate range, in the limit $\alpha \rightarrow \infty$, the results for the wavefucntions and the spectra are left unchanged. Indeed, the coordinate range of $z$ is now $-\pi / 2 \leq z \leq$ $\pi / 2$ and the eigenfunctions and eigenvalues turn out to be the same as the ones in (4.16) and (4.17).

Another case that is easy to solve occurs when $a \rightarrow 0$, which can be attained only in the sphere case. As can be seen from (3.16) we obtain the metric

$$
d s^{2}=2 d u d v+d x^{2}+d \vec{x}_{3}^{2}+d \vec{x}_{4}^{2}-J^{2}\left(4 x^{2}+\vec{x}_{4}^{2}\right) d u^{2} .
$$

Hence, three of the transverse scalars are massless and the others are massive with different masses. The corresponding frequencies are

$$
\omega_{(3)}= \pm|n| \quad \omega_{(x)}= \pm \sqrt{n^{2}+4 P_{+}^{2} J^{2}}, \quad \omega_{(4)}= \pm \sqrt{n^{2}+P_{+}^{2} J^{2}}
$$

with multiplicities, for each sign, three, one and four, respectively.

The fermionic spectrum on the other hand is very simple and completely independent of any limit taken. The frequencies, with multiplicity eight are

$$
\omega= \pm \sqrt{n^{2}+P_{+}^{2} J^{2}} .
$$

Finally, we briefly comment on the spectra for the PP-wave corresponding to the geodesic with $\theta=0$. The Schrödinger potentials appearing in the equation of the scalar fluctuations can be found in (3.27). They are all physical in the sense that they do not violate the unitarity bound $-\frac{1}{4} \frac{1}{x^{2}}$. In the limit $b \rightarrow \infty$ the potentials look much more tractable (3.31), however, also with this simplification we were not able to find the spectra of the fluctuations explicitly. The equations for the fermionic modes (4.10) with $f$ given in (3.32) turn out to be equally elusive.

\section{Disk distribution in the limit of large radius}

The fact that the metric (3.15) is well defined in the limit $a \rightarrow \infty$, suggests that there exists a corresponding limit of the background (3.18) before the Penrose limit is taken. Revealing the precise connection is the purpose of this section.

Let us take the limit $r_{0} \rightarrow \infty$ of the metric (3.18) which pushes the radius of the disk to infinity. The resulting geometry is well defined provided that we also approach $\theta=0$ or $\theta=\pi / 2$. It is straightforward to see that in the former case one obtains the metric 
for D3-branes smeared uniformly in two transverse directions and the harmonic function behaves as $1 / r^{2}$, where $r$ is the radius of the four-dimensional transverse subspace.

However, quite surprisingly, in the case that $\theta \rightarrow \pi / 2$ we obtain a distribution of D3branes that is uniformly smeared only over the half-plane. Indeed, consider the redefinitions

$$
r_{0} \rightarrow r_{0} R, \quad \theta=\frac{\pi}{2}-\frac{z}{r_{0} R}, \quad \phi \rightarrow \frac{x_{6}}{r_{0} R^{2}}, \quad x^{\mu} \rightarrow x^{\mu} R,
$$

followed by the limit $R \rightarrow \infty$. Then the metric takes the form

$$
d s^{2}=r\left(r^{2}+z^{2}\right)^{1 / 2} \eta_{\mu \nu} d x^{\mu} d x^{\nu}+\frac{1}{r_{0}^{2} r\left(r^{2}+z^{2}\right)^{1 / 2}}\left(\left(r^{2}+z^{2}\right)\left(d r^{2}+d z^{2}\right)+r_{0}^{2} d x_{6}^{2}+r^{2} z^{2} d \Omega_{3}^{2}\right) .
$$

The parameter $r_{0}$ can be absorbed by rescaling the coordinates appropriately, so that we can set $r_{0}=1$. In order to reveal the structure of the D3-brane distribution that we have advertised, consider another change of variables

$$
r=\sqrt{r_{5}-x_{5}}, \quad z=\sqrt{r_{5}+x_{5}} .
$$

Then the metric becomes

$$
d s^{2}=H^{-1 / 2} \eta_{\mu \nu} d x^{\mu} d x^{\nu}+H^{1 / 2} \sum_{i=1}^{6} d x_{i}^{2},
$$

where we have defined $x_{i}, i=1,2,3,4$ from $d r_{4}^{2}+r_{4}^{2} d \Omega_{3}^{2}$, where $r_{4}^{2}=r_{5}^{2}-x_{5}^{2}$. Therefore, naturally $r_{5}^{2}=\sum_{i=1}^{5} x_{i}^{2}$. The function $H$ is

$$
H=\frac{1}{2} \frac{1}{r_{5}\left(r_{5}-x_{5}\right)},
$$

and is indeed a harmonic function in the space spanned by $x_{1}, \ldots, x_{5}$. The distribution of D3-branes is obviously uniform in the $x_{6}$-direction. However, along the $x_{5}$-direction the distribution extends only along the positive axis, i.e. for $x_{5}>0$. This is in perfect agreement with the singularity of the harmonic function $H$ which is localized at $x_{1}=x_{2}=x_{3}=x_{4}=0$ and $x_{5}>0$. This can also be confirmed by a direct computation of the following integral that results when we consider the smearing of the D3-branes in the half plane

$$
\begin{aligned}
\int_{0}^{\infty} & d a \int_{-\infty}^{\infty} d b \frac{1}{\left[x_{1}^{2}+\cdots+x_{4}^{2}+\left(x_{5}-a\right)^{2}+\left(x_{6}-b\right)^{2}\right]^{2}} \\
& =\frac{\pi}{2} \int_{0}^{\infty} d a \frac{1}{\left[x_{1}^{2}+\cdots+x_{4}^{2}+\left(x_{5}-a\right)^{2}\right]^{3 / 2}} \\
& =\frac{\pi}{2} \frac{1}{r_{5}\left(r_{5}-x_{5}\right)} .
\end{aligned}
$$

Note that the limiting metric (5.2) (or equivalently (5.4)) exhibits a $\mathrm{SO}(4) \times \mathrm{SO}(2)$ symmetry like the original metric (3.18).

We also not that the limit (5.1) can also be applied to the non-extremal metric (3.1). However, the resulting metric is singular and it does not have a regular horizon. 


\subsection{PP-wave limit}

Similar to our previous examples consider the PP-wave limit around a geodesic situated at $z=0$. The corresponding three dimensional effective metric is of the general form (3.5) with $\alpha=x_{6}$ and non-zero components given by

$$
G_{t}=r^{2}, \quad G_{x_{6}}=\frac{1}{r^{2}}, \quad G_{r}=1 .
$$

After we multiply the metric by an overall $R^{2}$, followed by the rescalings $x^{\mu} \rightarrow x^{\mu} / R$ and $z \rightarrow z / R$ we get, in the limit $R \rightarrow \infty$, a metric of the form (3.13), where the functions are

$$
\begin{aligned}
& A_{x}=\frac{1}{r^{2}}-J^{2} r^{2}=-J \frac{\sin ^{2} 2 J u}{\cos 2 J u}, \\
& A_{3}=r^{2}=-\frac{1}{J} \cos 2 J u,
\end{aligned}
$$

Passing to the Brinkman coordinates we get a metric of the form (3.15 but with

$$
\begin{aligned}
& F_{x}=-J^{2}\left(1-\frac{3}{\cos ^{2} 2 J u}\right) \\
& F_{3}=-J^{2}\left(1+\frac{1}{\cos ^{2} 2 J u}\right) \\
& F_{4}=-J^{2}
\end{aligned}
$$

which is the $a \rightarrow \infty$ limit of (3.16).

\section{Concluding remarks}

In this paper we have constructed new families of PP-wave solutions of type-IIB string theory that have light-cone (LC)-time dependent five-form flux and metric, vanishing threeform fluxes and constant dilaton/axion. In general these backgrounds preserve sixteen supersymmetries and the world-sheet action has non-trivial interactions due to the LC time dependence. The latter implies that the string theory, at least in a generic background, is not exactly soluble. Furthermore, we studied fluctuations of the Green-Schwarz string in these backgrounds and were able to relate the relevant equations to supersymmetric quantum mechanics problems. Most importantly, this guarantees that the spectra have no tachyons and in some cases we were able to work them out exactly.

We have showed that there are special states in the perturbative string spectrum for particular values of the light-cone parameter $P_{+}$. This is somewhat reminiscent of the special discrete states in two-dimensional string theory that occur for a particular discrete set of values of the momentum [32. We also note that some of our PP-waves, by being simultaneously non-trivial and solvable, can be used to elucidate an important general issue, namely, whether a uniform light-cone gauge choice can be made. In that respect, we note that there are additional string states related to the folding of strings on themselves, which are not captured by the theory obtained if a uniform light-cone gauge choice is made (for original work on this issue, see [33]). It will be very interesting to pursue further these and related issues. 
A generic feature of our PP-waves is that some of the $F_{i i}$ components of the metric can blow up. It has been argued [29] that this leads to singular string propagation and therefore, naively, one might think that these solutions are unphysical. However, we believe that this is not the case. Note, that these solutions are Penrose limits of backgrounds used in the AdS/CFT correspondence to describe the deformation of $\mathcal{N}=4 \mathrm{SYM}$ by turning on expectation values of scalar fields. On the field theory side this is the simplest deformation one can think of and is by no means singular. Furthermore, in the IR the supergravity dual is not a valid description because the curvature blows up. This is a reflection of the fact that the deep IR is better described by a set of free abelian gauge theories, which do not have a "good" supergravity dual. Since the geodesics we used for the the Penrose limits probe precisely the region in the deep IR, it was to be expected that the string theory description might break down at some point and there should exist a valid field theory description. ${ }^{6}$ This field theory should be a truncation of $\mathcal{N}=4 \mathrm{SYM}$ as in [1] where the scalars now obtain vacuum expectation values in the form of continuous distributions. It would be interesting to investigate this issue further by performing a direct field theory computation. In particular, we suspect that the IR is described by a free field theory.

We should also mention that the Penrose limit of the non-extremal backgrounds washes out the finite temperature effects responsible for the existence of the horizon in the original background which is lost after the limit is taken. This has been observed also in previous work and is a consequence of the incompatibility of the existence of a covariantly constant null Killing vector with the presence of an event horizon [35].

\section{Acknowledgments}

A.B. would like to thank J. Gomis and N. Halmagyi for discussions. The work of A.B. was supported in part by the DOE under grant No. DE-FG03-92ER40701. K.S. would like to thank I. Bars for a usefull correspondence. He also acknowledges the financial support provided through the European Community's Human Potential Programme under contracts HPRN-CT-2000-00122 "Superstring Theory" and HPRN-CT-2000-00131 "Quantum Structure of Space-time", the support by the Greek State Scholarships Foundation under the contract IKYDA-2001/22 "Quantum Fields and Strings", as well as NATO support by a Collaborative Linkage Grant under the contract PST.CLG.978785 "Algebraic and Geometrical Aspects of Conformal Field Theories and Superstrings".

\section{References}

[1] D. Berenstein, J.M. Maldacena and H. Nastase, Strings in flat space and PP waves from $N=4$ super Yang-Mills, J. High Energy Phys. 04 (2002) 013 hep-th/0202021.

[2] R.R. Metsaev, Type IIB Green-Schwarz superstring in plane wave Ramond-Ramond background, Nucl. Phys. B 625 (2002) 70 hep-th/0112044.

\footnotetext{
${ }^{6} \mathrm{~A}$ breakdown of the string description was also observed in [34 for different backgrounds. In the PPwaves studied in [34] it was possible to switch to another dual string theory background, whereas in our case only field theory is a reliable description in the IR.
} 
[3] R.R. Metsaev and A.A. Tseytlin, Exactly solvable model of superstring in plane wave Ramond-Ramond background, Phys. Rev. D 65 (2002) 126004 hep-th/0202109.

[4] R. Penrose, Any space-time has a plane-wave as a limit, Differential Geometry and Relativity, Reidel, Dordrecht, 1976.

[5] M. Blau, J. Figueroa-O'Farrill, C. Hull and G. Papadopoulos, Penrose limits and maximal supersymmetry, Class. and Quant. Grav. 19 (2002) L87 hep-th/0201081.

[6] J.H. Schwarz and P.C. West, Symmetries and transformations of chiral $N=2 d=10$ supergravity, Phys. Lett. B 126 (1983) 301;

J.H. Schwarz, Covariant field equations of chiral $N=2 d=10$ supergravity, Nucl. Phys. B 226 (1983) 269;

P.S. Howe and P.C. West, The complete $N=2, d=10$ supergravity, Nucl. Phys. B 238 (1984) 181 .

[7] P.G.O. Freund and M.A. Rubin, Dynamics of dimensional reduction, Phys. Lett. B 97 (1980) 233 ;

B. Biran, F. Englert, B. de Wit and H. Nicolai, Gauged $N=8$ supergravity and its breaking from spontaneous compactification, Phys. Lett. B 124 (1983) 45, erratum ibid. B128 (1983) 461.

[8] M. Blau, J. Figueroa-O'Farrill, C. Hull and G. Papadopoulos, A new maximally supersymmetric background of IIB superstring theory, J. High Energy Phys. 01 (2002) 047 hep-th/0110242.

[9] K. Sfetsos, Gauging a nonsemisimple WZW model, Phys. Lett. B 324 (1994) 335 hep-th/9311010;

K. Sfetsos and A.A. Tseytlin, Four-dimensional plane wave string solutions with coset CFT description, Nucl. Phys. B 427 (1994) 245 hep-th/9404063.

[10] C.R. Nappi and E. Witten, $A$ WZW model based on a nonsemisimple group, Phys. Rev. Lett. 71 (1993) 3751 hep-th/9310112.

[11] R. Güven, Plane wave limits and t-duality, Phys. Lett. B 482 (2000) 255 hep-th/0005061.

[12] M. Cvetič, H. Lu and C.N. Pope, Penrose limits, PP-waves and deformed M2-branes, hep-th/0203082.

[13] R. Corrado, N. Halmagyi, K.D. Kennaway and N.P. Warner, Penrose limits of RG fixed points and PP-waves with background fluxes, hep-th/0205314.

[14] D. Brecher, C.V. Johnson, K.J. Lovis and R.C. Myers, Penrose limits, deformed PP-waves and the string duals of $N=1$ large- $N$ gauge theory, J. High Energy Phys. 10 (2002) 008 hep-th/0206045.

[15] I. Bena and R. Roiban, Supergravity PP-wave solutions with 28 and 24 supercharges, hep-th/0206195.

[16] J. Michelson, A PP-wave with 26 supercharges, Class. and Quant. Grav. 19 (2002) 5935 hep-th/0206204.

[17] R. Casero, Penrose limit of a non-supersymmetric rg fixed point, Nucl. Phys. B 649 (2003) 143 hep-th/0207221.

[18] E. Floratos and A. Kehagias, Penrose limits of orbifolds and orientifolds, J. High Energy Phys. 07 (2002) 031 hep-th/0203134. 
[19] J. Maldacena and L. Maoz, Strings on PP-waves and massive two dimensional field theories, J. High Energy Phys. 12 (2002) 046 hep-th/0207284.

[20] I. Bakas and J. Sonnenschein, On integrable models from PP-wave string backgrounds, Ð. High Energy Phys. 12 (2002) 049 hep-th/0211257.

[21] N. Kim, Comments on IIB PP-waves with Ramond-Ramond fluxes and massive two dimensional nonlinear sigma models, hep-th/0212017.

[22] M. Cvetič and D. Youm, Rotating intersecting m-branes, Nucl. Phys. B 499 (1997) 253 hep-th/9612229;

P. Kraus, F. Larsen and S.P. Trivedi, The coulomb branch of gauge theory from rotating branes, J. High Energy Phys. 03 (1999) 003 hep-th/9811120.

[23] J.G. Russo and K. Sfetsos, Rotating d3 branes and QCD in three dimensions, Adv. Theor. Math. Phys. 3 (1999) 131 hep-th/9901056.

[24] C. Csáki, Y. Oz, J. Russo and J. Terning, Large-N QCD from rotating branes, Phys. Rev. D 59 (1999) 065012 hep-th/9810186.

[25] A. Brandhuber and K. Sfetsos, Wilson loops from multicentre and rotating branes, mass gaps and phase structure in gauge theories, Adv. Theor. Math. Phys. 3 (1999) 851 hep-th/9906201.

[26] M. Blau, J. Figueroa-O'Farrill and G. Papadopoulos, Penrose limits, supergravity and brane dynamics, Class. and Quant. Grav. 19 (2002) 4753 hep-th/0202111.

[27] D.Z. Freedman, S.S. Gubser, K. Pilch and N.P. Warner, Continuous distributions of D3-branes and gauged supergravity, J. High Energy Phys. 07 (2000) 038 hep-th/9906194.

[28] A. Brandhuber and K. Sfetsos, Current correlators in the coulomb branch of $N=4$ sym, J. High Energy Phys. 12 (2000) 014 hep-th/0010048.

[29] G.T. Horowitz and A.R. Steif, Space-time singularities in string theory, Phys. Rev. Lett. 64 (1990) 260 .

[30] E. Witten, Dynamical breaking of supersymmetry, Nucl. Phys. B 188 (1981) 513.

[31] F. Cooper, A. Khare and U. Sukhatme, Supersymmetry and quantum mechanics, Phys. Rept. 251 (1995) 267 hep-th/9405029.

[32] I.R. Klebanov and A.M. Polyakov, Interaction of discrete states in two-dimensional string theory, Mod. Phys. Lett. A 6 (1991) 3273 hep-th/9109032.

[33] W.A. Bardeen, I. Bars, A.J. Hanson and R.D. Peccei, A study of the longitudinal kink modes of the string, Phys. Rev. D 13 (1976) 2364.

[34] E.G. Gimon, L.A. Pando Zayas and J. Sonnenschein, Penrose limits and RG flows, hep-th/0206033.

[35] V.E. Hubeny and M. Rangamani, No horizons in PP-waves, J. High Energy Phys. 11 (2002) 021 hep-th/0210234. 